\title{
Intelligente Zustandsüberwachung von Brückenbauwerken mit Hilfe faseroptischer Sensoren basierend auf der Rayleigh-Rückstreuung
}

\author{
Immanuel Roßteutscher, Philipp Wohlleben, Uwe Waizenhöfer \\ Institut für Sensor- und Aktortechnik \\ Am Hofbräuhaus $1 b$ \\ 96450 Coburg
}

\section{Zusammenfassung}

Allein in Bayern gibt es 10914 Brücken, von denen sich 538 in einem ungenügenden Zustand befinden [1]. Die Zahl der sanierungsbedürftigen Bauwerke wird in der Zukunft weiterhin steigen, da der zunehmende Schwerlastverkehr bei der Konzeptionierung sowie dem Bau der Verkehrsbrücken meist nicht berücksichtigt wurde. Die Begutachtung des Zustandes einer Brücke ist mit einem hohen Zeit- und Personalaufwand verbunden. Im Rahmen des Forschungsprojektes „i.bridge" wird für diese Problemstellung ein neuronales Netz entwickelt, welches mittels Sensoren verschiedene Parameter überwacht und somit eine Aussage über den Zustand einer Brücke liefern kann.

Für die sensorische Erfassung des Brückenzustandes durch Messung von Dehnung, Beschleunigung und Temperatur werden neben bewährten Technologien auch neuartige Sensortechnologien eingesetzt. Eine dieser Technologien basiert auf der Messung der Licht-Rückstreuung in einer optischen Faser mittels optischer Frequenzbereichsreflektometrie. Mit diesem Messprinzip können ortsaufgelöst die Dehnungswerte entlang der Glasfaser gemessen werden. Dadurch wird es möglich, Risse in der Betonstruktur eines Brückenbauwerks zu detektieren. Auch die Schwingungsmoden eines Brückenbauwerks können erfasst werden um Rückschlüsse auf den Zustand der Brücke zu geben. Der Vorteil dieses Systems, gegenüber herkömmlichen Dehnungsmessstreifen, liegt u.a. darin, dass lediglich eine optische Faser, die selbst als ortsaufgelöster Sensor fungiert, am Bauwerk installiert werden muss. Dadurch kann darauf verzichtet werden, eine Vielzahl von Einzelsensoren zu montieren, sowie die erforderlichen elektrischen Leitungen zu verlegen.

Es konnte gezeigt werden, dass eine ortsaufgelöste Erfassung der Durchbiegung und Schwingung des Brückenbauwerks mit einem faseroptischen Messsystem basierend auf der RayleighRückstreuung erfolgreich realisiert werden kann.

Keywords: Brücken-Monitoring, faseroptische Sensoren, Rayleigh-Rückstreuung, Neuronales Netz, Zustandsüberwachung

\section{Notwendigkeit der Zustandsüberwachung von Brückenbauwerken}

Als wichtigstes mitteleuropäisches Transitland steht Deutschland vor der Herausforderung, die Leistungsfähigkeit der Verkehrsinfrastruktur zu erhalten und kontinuierlich auszubauen. Neben dem Bau neuer bzw. dem Ausbau bestehender Trassen kommt dem Erhalt der Infrastruktur eine Schlüsselrolle zu. Neuralgische Bauwerke sind vor allem Brücken, da ihre Erhaltung bzw. ihr Ersatz enorme Investitionskosten zur Folge hat und damit volkwirtschaftlich in hohem Maße zu Buche schlägt.
Das Durchschnittsalter von Bahnbrücken beträgt bekanntlich ca. 80 Jahre und von Straßenbrücken i. M. ca. 40 Jahre. Vor dem Hintergrund des steigenden Verkehrsaufkommens und deutlich höherer Verkehrsgewichte (Gigaliner, Logistikoptimierung, etc.) und eines Brückenbestandes i. H. v. ca. 130.000 Brücken ist es daher erforderlich, ein kontinuierliches Monitoring zu ergänzen und es damit dem Fachpersonal zu erleichtern, Schäden zu erkennen. Die bisherige Überwachungsphilosophie zur Erkennung von Schäden, beruht auf zeit- und kostenintensiven Kontrollen in festen Zeitintervallen, welche fast ausschließlich durch Fachpersonal vor Ort erbracht werden. 
Grundsätzlich sind erhebliche Aufwendungen zur Gewährleistung und Aufrechterhaltung der Verfügbarkeit, Verkehrssicherheit und Lebensdauer für Brückenbauwerke erforderlich. Vor diesem Hintergrund ist es eminent, Schäden oder Schadenspotenziale möglichst frühzeitig zu erkennen.

In diesem Forschungsvorhaben wird der Ansatz verfolgt, moderne optische Sensoren zu verwenden, um das Brückenbauwerk in Echtzeit mittels eines innovativen neuronalen sensordatenverarbeitenden Systems zu überwachen. Im Gegensatz zur heutigen Technologie stehen hierbei nicht mehr einzelne Sensoren und deren Messgrößen im Fokus der Auswertung, sondern alle Sensordaten werden fusioniert und in einem entsprechend programmierten neuronalen Netzwerk verarbeitet. Durch eine derartige "intelligente" Brückenüberwachung wird es möglich, ein sich selbst konfigurierendes Überwachungssystem zu realisieren, welches den Betreiber detailliert über den Ist-Zustand des Brückenbauwerks informiert. Die zur Verfügung gestellten Daten informieren in Echtzeit über die Standsicherheit und erlauben damit Maßnahmen nutzungs- und verschleißabhängig und damit effizient und zum "richtigen Zeitpunkt" einzuleiten.

\section{Das OFDR - Messsystem auf Basis der Rayleighrückstreuung in optischen Fasern}

Die Rayleigh(rück-)streuung in einem Lichtwellenleiter entsteht zum größten Teil durch örtliche Änderungen der Dichte des Faserkerns. Mit der Dichteänderung verändert sich somit auch die Brechzahl innerhalb des Faserkerns. Diese Übergänge wirken als Streuzentren für die Rayleighstreuung. Dieser Effekt lässt sich durch Dotierung des Quarzglases mit Fremdatomen, z.B. Germaniumoxid $\left(\mathrm{GeO}_{2}\right)$, verstärken. Ein Teil des gestreuten Lichtes wird in Richtung der Lichtquelle zurückgestreut.

Handelsübliche Glasfasern werden heutzutage so dämpfungsarm wie möglich hergestellt. Trotz hochreinen Materialien reicht die Rayleighrückstreuung in solchen Fasern trotzdem aus, um diese zur Messung der Dehnung entlang der Faser zu verwenden.

Bei dem zur Brücken-Zustandsüberwachung verwendeten Messsystem „Optical Distributed Sensor Interrogator", kurz ODiSI, handelt es sich um ein System, welches auf Basis der optischen Reflektometrie arbeitet. In der optischen Reflektometrie existieren drei unterschiedliche Verfahren: Die Zeitbereichsreflektometrie OTDR (Optical time domain reflectometry), die optische Reflektometrie mit niedriger Kohärenz OLCR (Optical low coherence reflectometry) sowie die Frequenzbereichsreflektometrie OFDR (Optical frequency domain reflectometry) [2].

Die hier verwendete Frequenzbereichsreflektometrie (OFDR) ist bei den Kriterien Reichweite (bis zu 100m) sowie einer Auflösung im Millimeterbereich zwischen der OTDR und der OLCR einzuordnen [3].

Die optische Frequenzbereichsreflektometrie ist ein Messverfahren zur ortsaufgelösten Bestimmung des komplexen Reflexionskoeffizienten [4]. Das Verfahren OFDR basiert auf homodyner Interferometrie mittels eines durchstimmbaren, schmalbandigen Lasers. Der Lichtstrahl wird in ein Mach-ZehnderInterferometer eingekoppelt. Das Licht wird an einem Strahlteiler geteilt und durch einen Referenzarm sowie einen Messarm geleitet und letztendlich wieder rekombiniert (Abb. 1). Das dadurch entstandene Interferenzsignal wird schließlich mit Hilfe eines optischen Detektors aufgezeichnet.
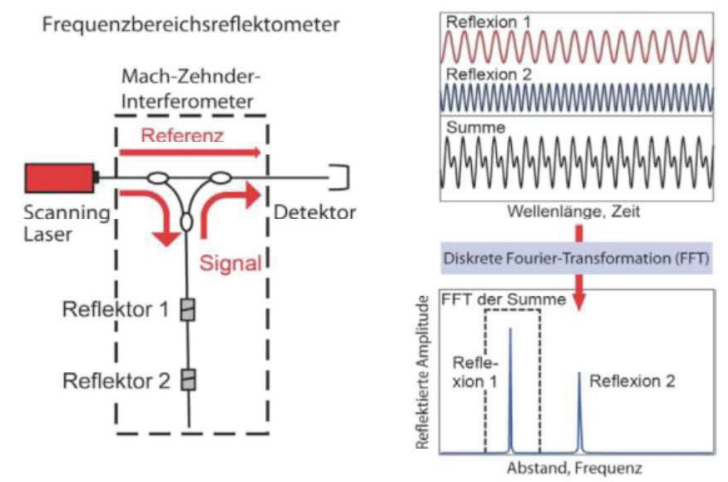

Abb.. 1: Schematischer Messaufbau des OFDR-Messsystems (links). Anschauliche Auswertung zweier Reflexionssignale. Die Amplituden sind ein Maß für die Stärke der Reflexion, die Frequenz hingegen lässt Rückschlüsse auf den Ort der Reflexion zu (rechts) [5].

Bei der Abtastung von Glasfasern mit OFDR ergibt sich für jede Faser ein charakteristisches Reflexionssignal, welches wie ein „Fingerabdruck", einzigartig ist. Das Reflexionssignal entsteht durch Rayleigh-Streuung an den Streuzentren (lokale Defekte, Brechzahlvariationen, Störung der Wellenleitergeometrie) innerhalb der Faser.

Wird die Faser durch äußere Einflüsse im Bereich von Fasersegmenten gedehnt (Dehnung durch Krafteinwirkung oder Dehnung durch Temperatur) wird das charakteristische 
Reflexionssignal an der entsprechenden Stelle ebenfalls gedehnt bzw. gestaucht. Das Maß der Dehnung kann durch eine Frequenzverschiebung $\Delta f$, welche proportional zu der einwirkenden Dehnung ist, erfasst werden [5].

\section{Daten zum Brückenbauwerk}

Bei der Brücke, die als Testobjekt dient, handelt es sich um eine $50 \mathrm{~m}$ lange Zweifeldbrücke über die Mainflut in der Nähe von Breitengüßbach, Bamberg. Die Brücke wurde im Jahre 1960 errichtet. Die Bundesstraße 279, die über diese Brücke führt, ist eine vielbefahrene Straße und verbindet Breitengüßbach mit Ebern. Das Verkehrsaufkommen liegt an Wochentagen bei etwa 15.000 25.000 Fahrzeugen.

Im Rahmen des Forschungsprojektes "i.bridge“ wurde von dem beteiligten Ingenieurbüro „Pötzl Ingenieure GmbH“ ein Berechnungsmodell erstellt um das Schwingungs-verhalten der Brücke zu simulieren.

Je nach verwendetem Modell (Stabwerkmodell bzw. Flächentragwerkmodell) wurde eine erste Eigenfrequenz von $4,175 \mathrm{~Hz}$ bzw. $4,183 \mathrm{~Hz}$ ermittelt (Abb. 2). Weiterhin wurde eine erwartete Dehnung von ungefähr $30 \mu \mathrm{m} / \mathrm{m}$ bei einer LKW-Überfahrt (40t) ermittelt.

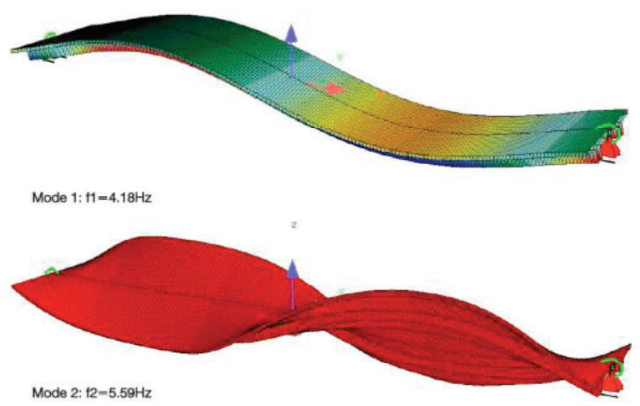

Abb.. 2: Stabwerkmodell: Berechnete Eigenfrequenzen sowie Eigenformen der ersten und zweiten Schwingungsmode bei $f 1 \approx 4,18 \mathrm{~Hz}$ und $\mathrm{f} 2 \approx 5,59$ $\mathrm{Hz}$.

\section{Montage der Faser am Brückenbauwerk}

Zur Aufnahme von Messdaten wurde eine optische Faser mit einer Länge von $5 \mathrm{~m}$ an der Unterseite eines Brückenträgers längs der Fahrtrichtung montiert (Abb. 3). Vor der Anbringung wurden Unebenheiten der Betonoberfläche durch Schleifen entfernt. Anschließend wurde die Oberfläche gereinigt. Zum Verschließen von Poren wurde - wie auch in der Messtechnik mit Dehnungsmessstreifen üblich - eine Versiegelung aufgebracht. Die Faser wurde unter einer geringen Vorspannung auf dem präparierten Beton verklebt und versiegelt.

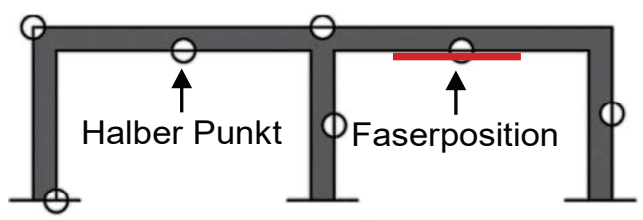

Abb.. 3: Schematische Darstellung des Anbringungsortes der Faser an der Brücke. Die Faser befindet sich mittig zwischen Nullpunkt und Mittelpfeiler.

\section{Detektion von Überfahrt-Ereignissen}

Das Messsystem liefert die ortsaufgelösten Dehnungswerte von ca. 1900 äquidistant verteilten Datenpunkten entlang einer $5 \mathrm{~m}$ Glasfaser. Bei der Mittelung über alle Faserpunkte erhält man - wie bei Einpunktsensoren - Dehnungswerte im zeitlichen Verlauf. Diese Werte geben während einer Kraftfahrzeugüberfahrt Aufschluss über die Durchbiegung und Schwingung der Brücke und liefern zudem Informationen über die Masse und Geschwindigkeit des überfahrenden Kraftfahrzeuges. Abb. 4 zeigt, dass anhand der Stärke der Dehnung der Brücke direkt zwischen der Überfahrt eines PKWs und eines LKWs unterscheiden werden kann. Zusätzlich lassen sich diese Informationen für eine Verkehrszählung nutzen.

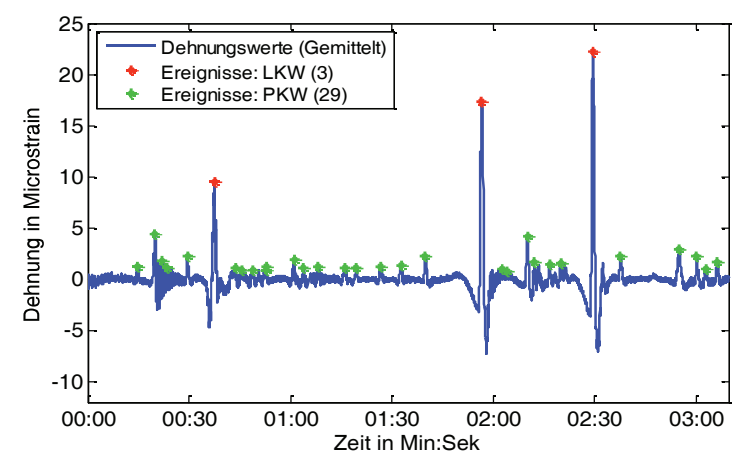

Abb. 4: Gemittelte Dehnungswerte im zeitlichen Verlauf. Aufgezeichnet wurden drei LKW- sowie 29 PKWÜberfahrten.

In Abb. 5 sind die Dehnungswerte der Überfahrten aus Abb. 4 ortsaufgelöst und farbcodiert aufgetragen. Deutlich zu erkennen sind die durch LKW-Überfahrten ausgelösten Bereiche mit hohen Dehnungen. Die Dehnungsmaxima bei der Faserposition $0 \mathrm{~m}$ treten im zeitlichen Verlauf ca. 0,2 Sekunden später auf als bei Faserposition $5 \mathrm{~m}$. Grund dafür ist die mit der Fortbewegung der Fahrzeuge verbundene dynamische Änderung der Durchbiegung der Brücke. 


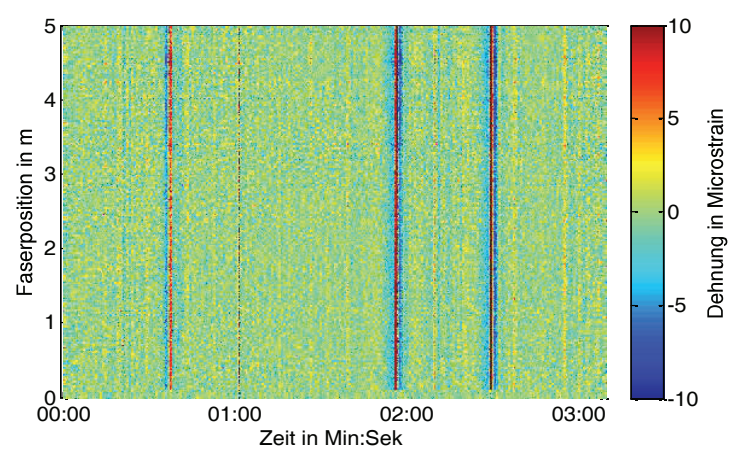

Abb. 5: Ortsaufgelöste Dehnungswerte als 2D-Graph farbcodiert dargestellt. Die horizontale Achse stellt die Zeit und die vertikale Achse die Position des Messpunktes entlang der Glasfaser dar. Die Farbcodierung informiert über die Stärke der Dehnung an den jeweiligen Faserpunkten im zeitlichen Verlauf.

\section{Analyse einer einzelnen Überfahrt}

Künftig sollen mit Hilfe eines neuronalen Netzes u.a. aus den gemessenen Dehnungswerten Informationen zum Brückenzustand abgeleitet werden können. Beispielsweise könnten Änderungen der Eigenfrequenzen, der Durchbiegung oder des Ausschwingverhaltens des Brückenbauwerks ein Indiz für eine Schädigung sein. Nachfolgend wird deshalb eine einzelne Überfahrt genauer Betrachtet.

Bei der Betrachtung eines Einzelereignisses (KFZ-Überfahrt) wird im Folgenden das Zeitsignal, das Frequenzspektrum, die Anregung der Eigenfrequenzen sowie das Abklingverhalten betrachtet. In Abb. 6 ist der zeitliche Verlauf der gemittelten Dehnungswerte aufgetragen. Die niederfrequente Durchbiegung der Brücke ist deutlich zu erkennen.

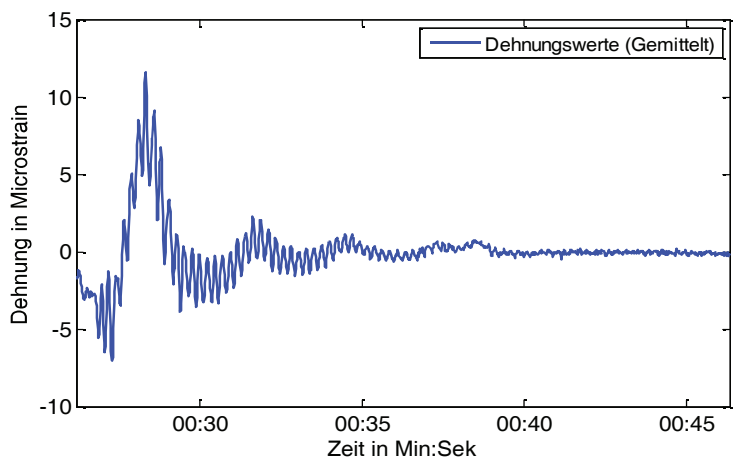

Abb. 6: Dehnungswerte eines Einzelereignisses im zeitlichen Verlauf.

Zusätzlich treten höherfrequente Dehnungsänderungen auf. Hierbei handelt es sich um die Eigenfrequenzen des Brückenbauwerks, mit der die Brücke - angeregt durch die Überfahrt des Fahrzeugs - schwingt. Dass es sich dabei tatsächlich um die Eigenfrequenzen des Brückenbauwerks handelt, wird durch das zugehörige Frequenzspektrum bestätigt (Abb. 7). Die markanten Peaks bei $4,25 \mathrm{~Hz}$ sowie $6,01 \mathrm{~Hz}$ entsprechen in guter Näherung der ersten und zweiten Eigenfrequenz des Berechnungsmodells. Der Peak bei $0,30 \mathrm{~Hz}$ kann der Durchbiegung der Brücke unter Lasteinfluss zugeordnet werden.

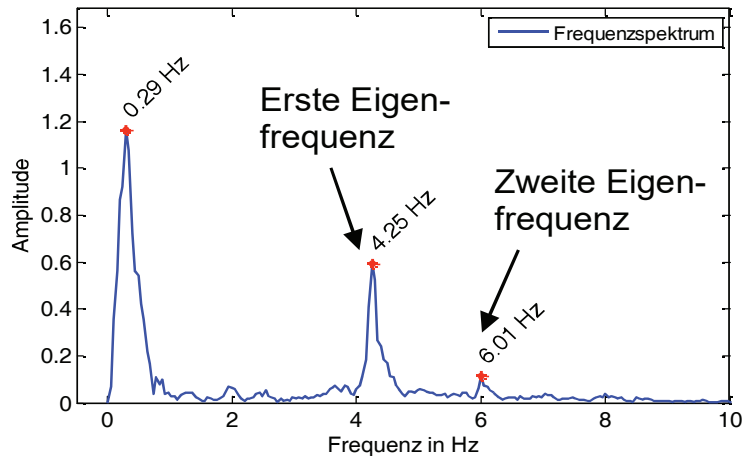

Abb. 7: Frequenzspektrum des Einzelereignisses aus Abb. 6.

Die ersten beiden Eigenfrequenzen werden direkt beim Auffahren des KFZs auf die Brücke angeregt. Während die zweite Eigenfrequenz zeitlich relativ schnell abklingt, ist nach ca. 45 Sekunden, nachdem das Fahrzeug die Brücke verlassen hat, noch eine Schwingung der ersten Eigenfrequenz messbar (Abb. 8).

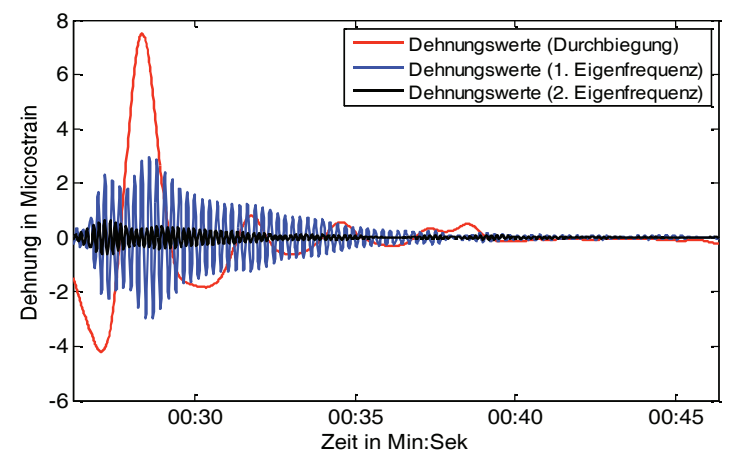

Abb. 8: Das Dehnungssignal aus Abb. 6 ist in dieser Darstellung jeweils mit einem Bandpassfilter im Bereich der Eigenfrequenzen bzw. Frequenz der Durchbiegung gefiltert.

Dem Ausschwingen der ersten Eigenfrequenz wird eine lineare Dämpfung zugrunde gelegt. Basierend auf den Dehnungswerten im Zeitverlauf während des Ausschwingens kann die Einhüllende berechnet und eine Parameterschätzung für eine lineare Dämpfung 
vorgenommen werden. Die Ergebnisse hierzu sind in Abb. 9 dargestellt.

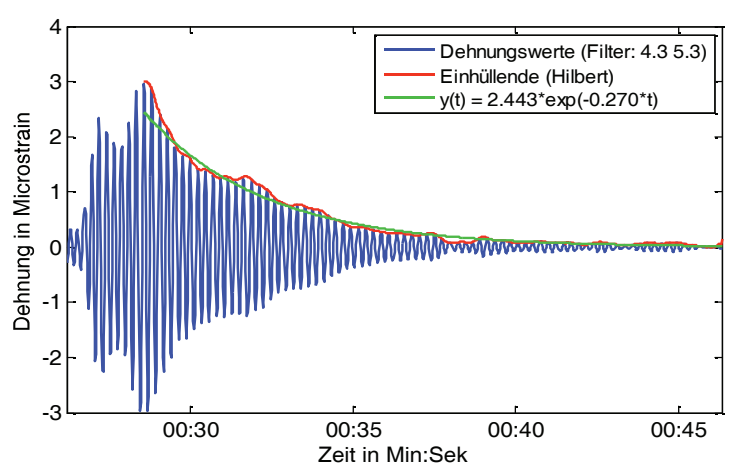

Abb. 9: Dehnungswerte des Ausschwingens der ersten Eigenfrequenz einschließlich der Einhüllenden und Parameterschätzung einer linearen Dämpfung.

Die Betrachtung des Einzelereignisses zeigt, dass sowohl die Durchbiegung der Brücke als auch die Schwingung der ersten beiden Eigenfrequenzen einschließlich dem Abklingverhalten mit dem faseroptischen Messsystem erfasst werden können.

\section{Gegenüberstellung der Rayleighfaser mit Dehnmessstreifen}

Für die Validierung der ermittelten Daten wurden neben den Messungen mit dem OFDR-Messsystem zusätzlich Messungen mit Dehn-messstreifen durchgeführt. Der verwendete Dehnmessstreifen hat eine Länge von $0,10 \mathrm{~m}$ und wurde direkt neben dem Mittelpunkt der 5m-Faser am Brückenbauwerk angebracht. In den Abb. 10 und 11 sind die Messsignale des DMS sowie der Glasfaser (gemittelt über $5 \mathrm{~m}$ ) gegenübergestellt. Die Amplituden der Dehnungswerte stimmen im Rahmen der Messgenauigkeit überein.

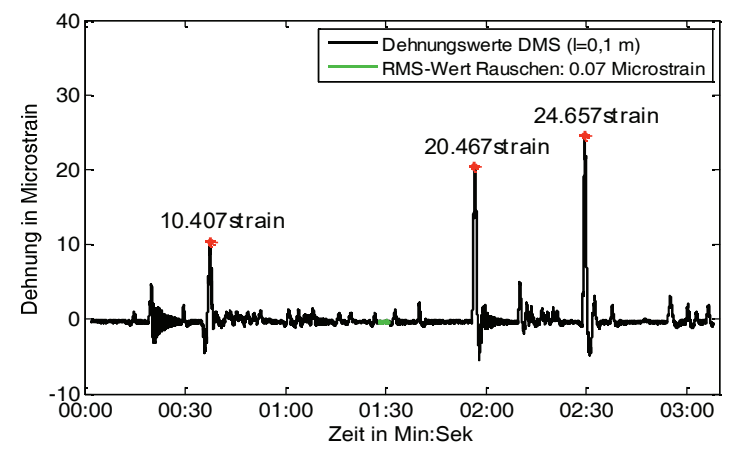

Abb. 10: Dehnungswerte im Zeitverlauf aufgenommen durch einen Dehnmessstreifen.

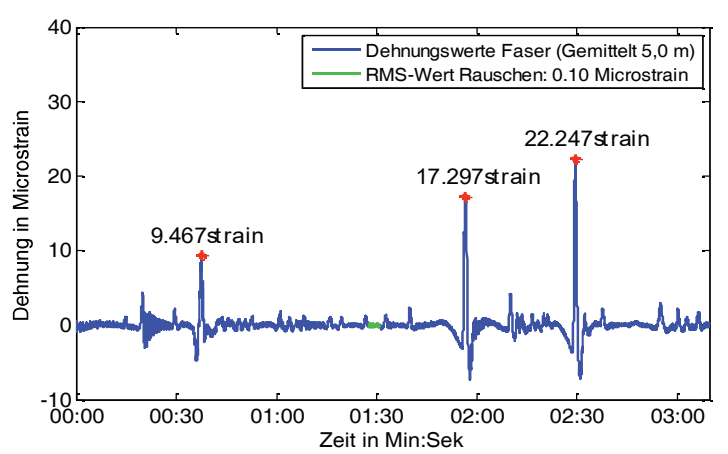

Abb. 11: Gemittelte Dehnungswerte im Zeitverlauf des Rayleigh-Systems, gemittelt über $5,0 \mathrm{~m}$.

Für einen direkten Vergleich bzw. des Rauschens der Messdaten wurde jeweils der quadratische Mittelwert (RMS-Wert) einer kurzen Zeitspanne mit Rauschen bestimmt. Die ermittelten Werte sind für verschiedene Mittelungslängen in der nachfolgenden Tab. 1 dargestellt:

Tab. 1: Vergleich des DMS-Sensors mit Daten des OFDR-Messsystems, basierend auf verschiedenen Mittelungslängen

\begin{tabular}{|l|c|c|c|c|}
\hline Sensor: & DMS & \multicolumn{3}{|c|}{ LWL } \\
\hline $\begin{array}{l}\text { Länge } \\
\text { örtlicher } \\
\text { Mittelung: }\end{array}$ & $/$ & $0,1 \mathrm{~m}$ & $1,0 \mathrm{~m}$ & $5,0 \mathrm{~m}$ \\
\hline $\begin{array}{l}\text { Daten- } \\
\text { punkte: }\end{array}$ & 1 & 38 & 382 & 1910 \\
\hline $\begin{array}{l}\text { Rauschen } \\
\text { (RMS-Wert): }\end{array}$ & $\begin{array}{c}0,07 \\
\mu \mathrm{m} / \mathrm{m}\end{array}$ & $\begin{array}{c}0,66 \\
\mu \mathrm{m} / \mathrm{m}\end{array}$ & $\begin{array}{c}0,18 \\
\mu \mathrm{m} / \mathrm{m}\end{array}$ & $\begin{array}{c}0,10 \\
\mu \mathrm{m} / \mathrm{m}\end{array}$ \\
\hline
\end{tabular}

\section{Dehnungsgradient des Brückenbauwerks bei LKW-Überfahrten}

Um den Dehnungsgradienten entlang des Brückenbauwerks bei Überfahrt-Ereignissen zu veranschaulichen, wurde eine $20 \mathrm{~m}$ lange Faser entlang der Fahrtrichtung an der Unterseite der Brücke angebracht. Die Faser erstreckt sich vom Nullpunkt der Brücke bis fast zum Mittelpfeiler.

Abb. 12 zeigt die ortsaufgelösten Dehnungswerte während der Überfahrt dreier LKWs im zeitlichen Verlauf. Es wird deutlich, dass die Dehnungswerte am „Halben Punkt" der Brücke (Faserposition $10 \mathrm{~m}$ ) am größten sind. Der Halbe Punkt befindet sich mittig zwischen Nullpunkt und Mittelpfeiler. An dieser Stelle ist die Durchbiegung bei Überfahrt-Ereignissen maximal. In Richtung Nullpunkt verlieren die Dehnungswerte an Intensität. Es entsteht ein Dehnungsgradient entlang der Brücke. 


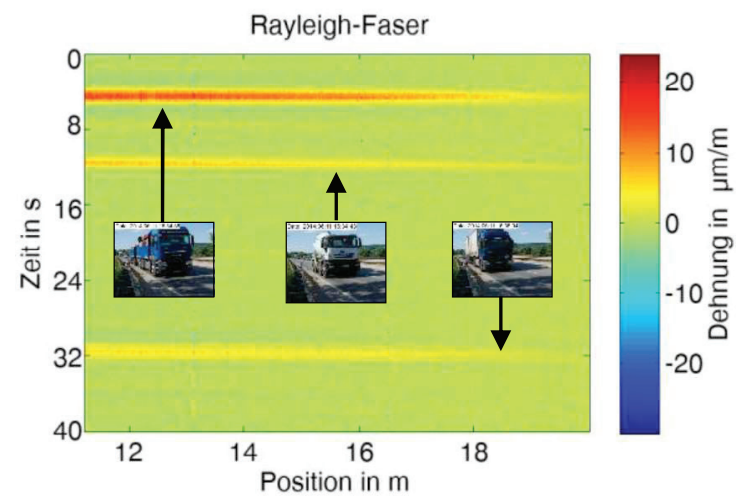

Abb. 12: Die ortsaufgelöste Durchbiegung der Brücke zeigt einen deutlichen Dehnungsgradienten.

Durch eine derart exakt ortsaufgelöste Dehnungs- und Schwingungsmessung des Brückenbauwerks können hilfreiche Informationen zum Zustand der Brücke gesammelt werden. Auch eine Detektion von Rissen im Beton (Ort und Stärke des Risses) ist möglich, und konnte unter Laborbedingungen bereits erfolgreich getestet werden.

\section{Literaturnachweis}

[1] Bundesregierung, „Antwort auf die kleine Anfrage zum Zustand der Straßenbrücken in Bayern - Drucksache 18/5523," Berlin, 2015

[2] Soller, B.J, Gifford, D.K., Wolfe, M.S. und Froggatt, M.E., „High resolution optical frequency domain reflectometry for characterization of components and assemblies," Optics Express (13), S. 666-674, 2005. doi: 10.1364/OPEX.13.000666

[3] Weid, J. P. von, Passy, R., Mussi, G. und Gisin, $\mathrm{N}$. „On the characterization of optical fiber network components with optical frequency domain reflectometry," Journal of Lightwave Technology (15), S. 1131-1141, 1997. doi: 10.1109/50.596958

[4] Müller, J., Charakterisierung integriert-optischer Silizium-Wellenleiter, München: Verlag Dr. Hut, 2013. doi: 10.15480/882.1123

[5] Samiec, D., „Verteilte faseroptische Temperatur- und Dehnungsmessung mit sehr hoher Ortsauflösung," Photonik (6), S. 34-37, 2011 